\title{
Endothelial and Autonomic Assessments in the Management of COVID-19
}

\author{
Albert Maarek \\ Research and Development, LD Technology, Miami, USA \\ Email: albert.ldteck@gmail.com
}

How to cite this paper: Maarek, A. (2021) Endothelial and Autonomic Assessments in the Management of COVID-19. Open Journal of Preventive Medicine, 11, 425-438. https://doi.org/10.4236/ojpm.2021.1112033

Received: October 19, 2021

Accepted: December 20, 2021

Published: December 23, 2021

Copyright (C) 2021 by author(s) and Scientific Research Publishing Inc. This work is licensed under the Creative Commons Attribution International License (CC BY 4.0).

http://creativecommons.org/licenses/by/4.0/ (c) (i) Open Access

\begin{abstract}
Background: The goal of this peer review is to outline the benefits of the TM Flow system and Lifeprobes Kiosk assessing the endothelial and autonomic system functions in the management of COVID-19 for triage of the patients at risk and diagnostic of autonomic nervous system dysfunction to understand the long COVID symptoms. Material and Methods: The TM Flow system is a non-invasive medical device that relies on pulse oximetry, blood pressure measurements, and very low electrical current stimulation of the skin via disposable electrodes to provide instantaneous assessments of patients' vital signs, endothelial function, sudomotor and autonomic nervous system functions, and ankle brachial index measurements. The Lifeprobes Kiosk performs automated pre-consultation patient assessments via an online patient check-in form and medical questionnaire, as well as determines the patient's vital signs, endothelial function markers and estimated body composition. COVID-19: Current Scientific Data: In the majority of cases, COVID-19 infection will manifest through mild to moderate symptoms (such as cough, runny nose, sore throat, headache, muscle aches, and fever) which would resolve without the need for special treatment or hospitalization. On the other hand, older individuals and those who suffer from underlying medical conditions-such as obesity, diabetes, cardiovascular disease, chronic respiratory disease, and/or cancer-are more susceptible to developing serious illness, often with a fatal outcome. Available evidence further indicates that COVID-19 vaccines reduce the number of new cases, while also lessening the risk of complications that would require hospitalization. However, these findings do not apply to the new delta variant, which leads to more severe complications in younger people than previous variants, even in those who have been vaccinated and/or do not have any underlying medical problems. Research conducted to date indicates that COVID-19 complications result from endothelial dysfunction or damage. In addition, COVID19 patients are increasingly reporting a wide range of debilitating conditions long after they have tested negative for the presence of the virus, giving rise to
\end{abstract}


the term "Long COVID" which has been attributed to autonomic nervous system dysfunction and small fiber neuropathy. TM Flow markers have been investigated by several clinical studies in the US, India, and Brazil. The clinical studies showed the relevance of the TM Flow endothelial and autonomic nervous markers. Conclusion: Findings yielded by peer-reviewed clinical studies confirm that the TM Flow system and Lifeprobes Kiosk are beneficial and effective non-invasive tools that provide immediate results. Therefore, both should be employed for the triage of COVID-19 patients to expediently identify those at risk of complications, as this would reduce the hospitalization and mortality rates. The TM Flow system is also capable of diagnosing "Long COVID" symptoms related to the autonomic nervous system and small fiber neuropathy.

\section{Keywords}

Endothelial Function, Autonomic Nervous System, COVID-19

\section{Introduction}

The SARS-CoV-2 (COVID-19) is the first virus in recorded history that has had prolonged and devastating effects on public health as well as economy on the global scale [1]. While it was initially believed that it would be contained within China, its rapid spread prompted the World Health Organization (WHO) to declare COVID-19 a Public Health Emergency of International Concern (PHEIC) on January $30^{\text {th }}, 2020$ [2]. Although a significant majority of people infected with the SARS-CoV-2 virus experience either no symptoms or suffer mild to moderate ailments, in some cases, the illness can be fatal or have long-lasting adverse consequences. The initial variants of this virus posed the greatest threat to older individuals and those with underlying medical conditions, such as obesity, diabetes, cardiovascular disease, chronic respiratory disease, and/or cancer [3] [4]. However, as the virus mutated, it became more invasive, whereby now even very young and healthy individuals can suffer severe issues. While several SARS-CoV-2 vaccines have been developed since the initial outbreak, their unequal availability across the globe limits their potential to curb the pandemic. Nonetheless, in countries with a high percentage of vaccinated eligible individuals, a steady decline in COVID-19 cases, hospital admissions, and death rates has been observed [5]. The most recent increases in COVID-19 cases have been attributed to a new variant of SARS-CoV-2, known as the "delta variant" which has a $60 \%$ higher transmission rate than the alpha variant and, according to the $\mathrm{CDC}$, has a basic reproduction rate of 5 - 8 [5]. This evidence, combined with the fact that currently available vaccines are less effective against all more recent variants relative to the alpha variant, suggests that further research into treatments offered to COVID-19 patients is warranted [5].

Given that endothelial dysfunction plays a key role in the pathogenesis of 
COVID-19 by disrupting the vitality of vessels, causing a pro-coagulative state, inflammation of the endothelium, and enabling leukocyte penetration [6] [7], and that the growing number of COVID-19 patients are presenting new symptom patterns long after they have tested negative for COVID-19 [8] [9], more effective means of preventing the infection and treating its long-term effects are necessary.

The TM Flow system and Lifeprobes Kiosk are valuable contributions to this endeavor, as they allow the Autonomic Nervous System (ANS) and endothelial function to be assessed in real-time, while providing instantaneous and reliable results, as discussed in the sections following.

\section{Material and Methods}

The TM Flow is a Medical Device Data System (MDDS) managing three hardware components US FDA cleared and EC Mark. The intended uses of the three components are reimbursable by most insurance companies according to the medical necessity of the testing. TM Flow can be used only on adult patients with very few contraindications such as patients undergoing external defibrillation, Arterio-Venous (AV) fistula or shunt, and bilateral mastectomy.

It has been successfully marketed in the USA for the early detection of chronic disease complications and chronic care management. It provides non-invasive tests that take 7 - 10 minutes to complete and yield immediate results. As shown in Figure 1, the TM Flow system comprises of three hardware components-LDOXI, SweatC, and TBL-ABI.

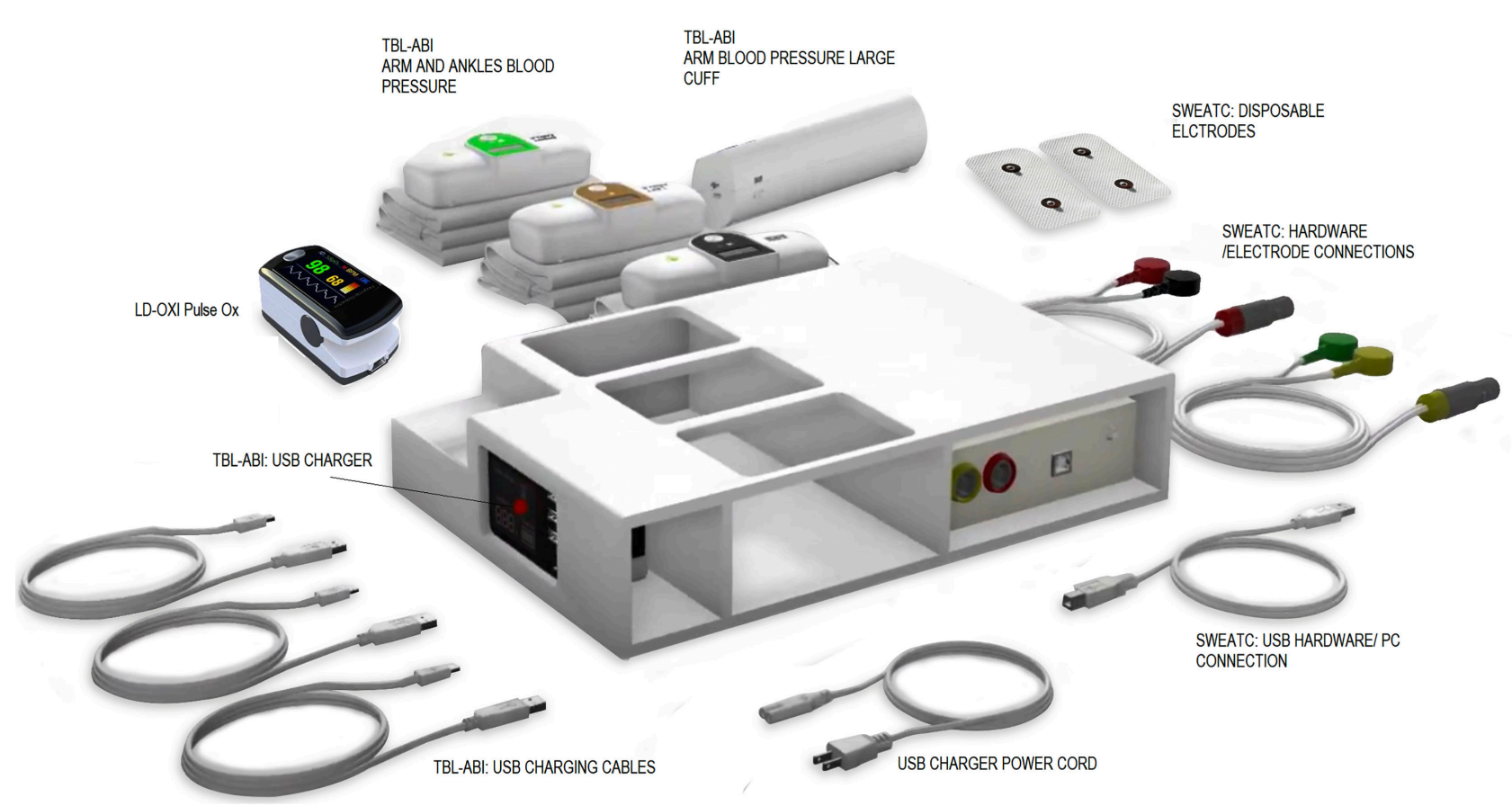

Figure 1. Image of the TM Flow system components. 
1) The LD-OXI allows for $\mathrm{SpO}_{2} \%$ and photoplethysmography (PTG/PPG) analysis in both time and frequency domain. It not only detects the heart rate but also provides markers related to the endothelium function. It also conducts Heart Rate Variability (HRV) analysis to determine the early onset of autonomic dysfunction using time domain analysis and frequency domain analysis, as well as cardiac autonomic reflex (also known as Ewing) tests-Valsalva maneuver, deep breathing, and postural change-to assess for cardiovagal denervation and/or autonomic nervous system failure.

2) The SweatC is LD Technology's proprietary medical device hardware that employs galvanic skin response technology to assess the sudomotor or sweat gland function. By using disposable electrodes placed on the feet, the SweatC delivers a predetermined electrical stimulation to the feet within a specified sequence. The resulting Sympathetic Skin Response (SSR) measurements are indicative of the patient's sudomotor or sweat gland function.

3) The TBL-ABI is a medical device that provides fast and accurate measurements of ankle brachial blood pressure, ankle brachial indices, and pulse volume plethysmography in adult patients. It is presently used to assess at risk of developing peripheral vascular disease or Peripheral Artery Disease (PAD).

As shown in Figure 2, the Lifeprobes Kiosk is a MDDS managing the data of five hardware components: LD-OXI, body temperature sensor, height and weight sensors, ES-BC (which estimates the body composition using bioimpedance analysis), and BP-BT (allowing for a self-administered, automated, blood pressure measurement). The Lifeprobes test is rapid and non-invasive and provides the results within two minutes of its initiation.

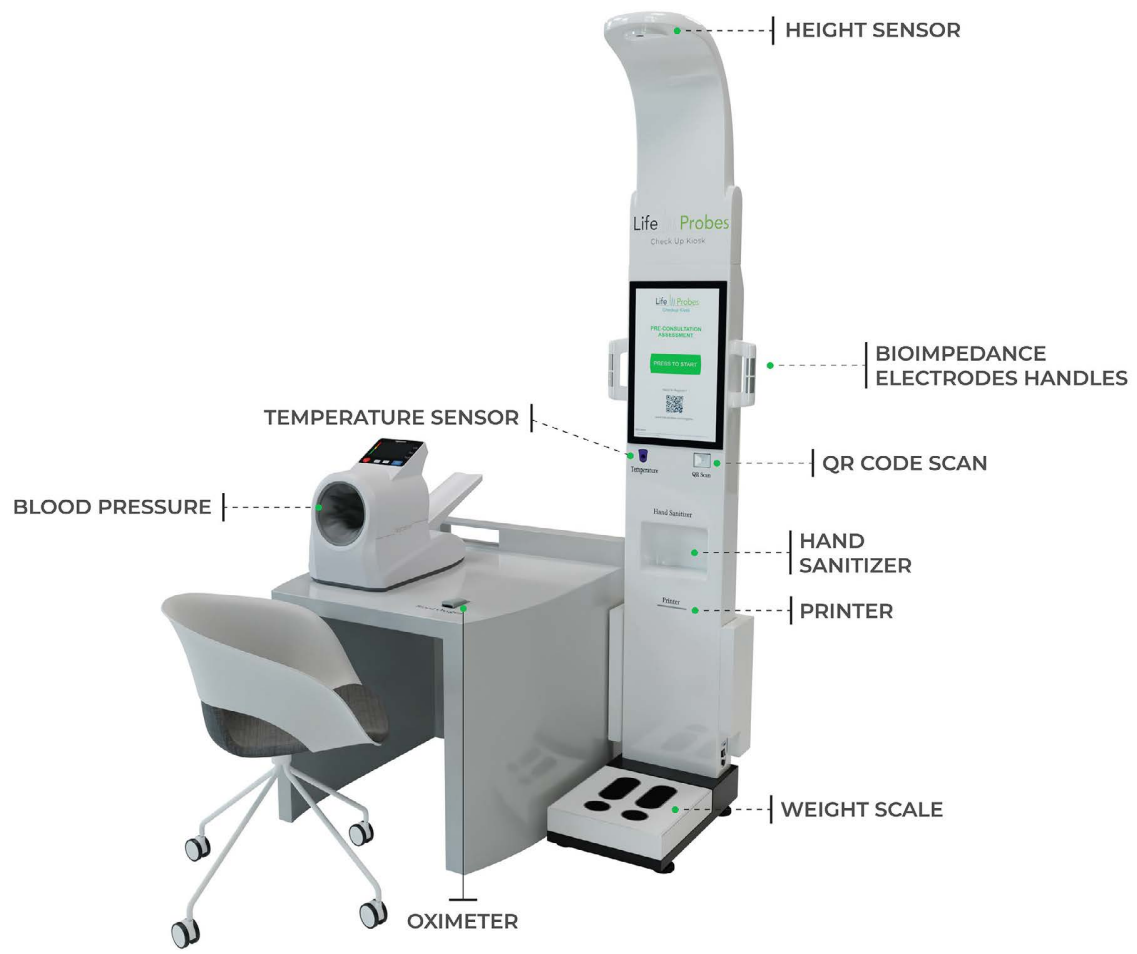

Figure 2. Image of the Lifeprobes kiosk components. 


\subsection{LD-OXI Plethysmography Analysis}

Once LD-OXI analysis is complete, the photoplethysmography (PTG or PPG) values displayed by the oximeter are converted using a novel algorithm (incorporating the first and second derivative) adapted from the work done by Elgendi [10].

The first derivative is utilized to calculate RR intervals (which refers to the time change between two successive R waves of the QRS complex as seen on an EKG), whereas arterial tone is estimated from the second derivative related to the SDPTG marker [11], as shown in Figure 3.

As can be seen from the graph, SDPTG denotes that the ratio of the height of $\mathrm{b} / \mathrm{a}$ decreases and $\mathrm{d} / \mathrm{a}$ increases in abnormal arterial stiffness. Once these measurements have been established, the first derivative of the photoplethysmography reading is converted into three main frequencies using the Fast Fourier Transforms to obtain the PTG spectral analysis [12], as shown in Figure 4.

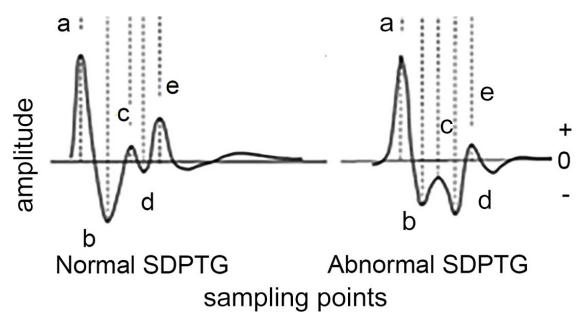

Figure 3. The changes of the SDPTG (namely "a", "b", “c”, “d”, and "e"). The height of each wave is measured from the baseline (0), with the values above the baseline being positive and those under it negative. The ratios of the height of each wave to the a-wave (b/a, c/a, d/a, and e/a) are used for wave analysis. The picture shows a normal and abnormal pattern.

Original PTG

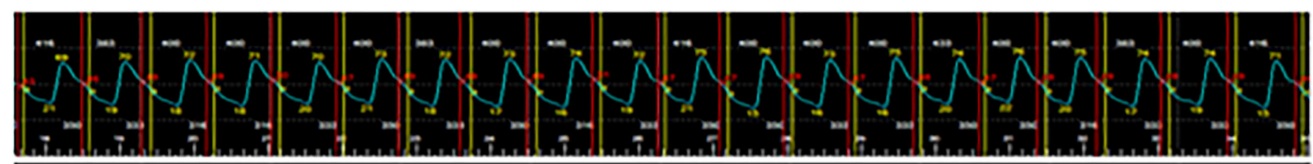

First derivative PTG Analysis: Heart Rate and RR intervals detection

Second derivative PTG Analysis: Arterial Tone Marker (SDPTG)

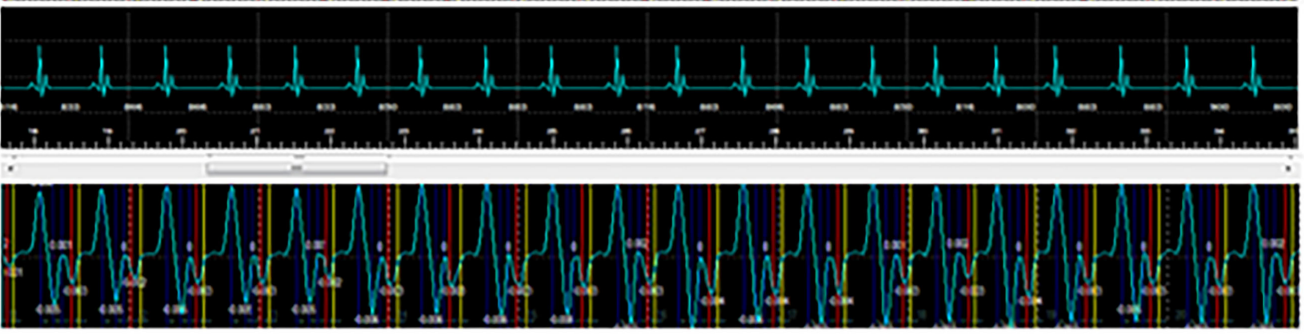

Fast Fourier transforms. Spectral analysis : Endothelial Markers

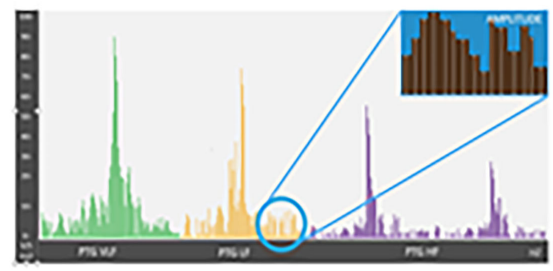

\section{STRESS INDEX=}

It is the amplitude of PTG VLF

\section{PTG VLF INDEX (PTGVLFI) =}

It is the amplitude of VLF divided by the NO Peak of the GSR measurement the PTG spectral Analysis
PTG INDEX (PTGI)=

It is the sum of amplitude between the Peaks of the Spectral analysis.

Figure 4. The steps involved in the conversion of the photoplethysmography (PTG) measurements and markers. 
As noted above, the first derivative analysis allows for the detection of RR intervals [10], whereas the second derivative provides the SDPTG marker correlated with the arterial stiffness [12]. Consequently, the spectral analysis yields the following endothelial markers:

- The Stress Index which is correlated with the CPR lab test findings [13];

- The PTG VLF Index which is correlated with the fibrinogen lab test results [13];

- The PTG Index capable of detecting coronary artery disease with high sensitivity and specificity [14];

- The PTG Total Power capable of detecting insulin resistance (indicative of endothelial cells function [15]) with high sensitivity and specificity [16].

\subsection{Endothelial Function Assessment}

The TM Flow and Lifeprobes Kiosk rely on five patented markers depicted in Figure 3 and Figure 4 to assess the endothelial function, whereby the diagnosis is guided by the number of borderline and abnormal results.

For example, for the early diagnosis of endothelial dysfunction, only one abnormal result is implied, whereas defined endothelial function is confirmed by the presence of two or three abnormal test results, and four or five abnormal results are indicative of severe endothelial dysfunction.

\subsection{Cardiac Autonomic Neuropathy (CAN) Assessment}

The TM Flow cardiac autonomic neuropathy assessment requires measurement of two Heart Rate Variability (HRV) test markers-Total Power (HRV frequency domain marker) and SDANN (HRV time domain marker) - and five Cardiac Autonomic Reflex Test (CART) markers, namely Valsalva ratio (RR interval changes during the Valsalva test), E/I ratio ( $R R$ interval changes during the deep breathing test), $\mathrm{K} 30 / 15$ ratio ( $\mathrm{RR}$ interval changes during the change in posture test), systolic pressure difference between lying down and standing up position, and diastolic pressure difference.

According to the CAN Subcommittee of the Toronto Consensus Panel criteria for CAN definition and severity, only one abnormal CART result is sufficient for the early CAN diagnosis, whereas two or three abnormal tests are indicative of definite CAN, and severe CAN should be suspected in the presence of Orthostatic Hypotension (OH) [17] [18] [19]. Available evidence further indicates that cardiac autonomic neuropathy is a marker of hypoglycemia risk [20] and can be evaluated to target the $\mathrm{Hb} 1 \mathrm{Ac}$ in diabetic patients undergoing treatment, as it increases the incidence of cardiac events by 3 - to 4 -fold [21].

\subsection{SweatC Patented Sudomotor Assessment}

During tests, SweatC sends a weak DC current to tactile disposable electrodes in order to stimulate the post-ganglionic cholinergic fibers (C-fibers), resulting in a cholinergic sympathetic stimulation and microcirculatory response which in- 
duce a release of sweat, allowing both phenomena to be captured and converted into electric potential (expressed in $\mathrm{mV}$ ). Based on the established criteria, a microcirculatory response value below $786 \mathrm{mV}$ and Sweat Peak response below 836 $\mathrm{mV}$ and above $512 \mathrm{mV}$ is indicative of reduced post-ganglionic cholinergic fibers (C-fibers) stimulation. Moreover, Sweat Peak response values below $512 \mathrm{mV}$ are a sign of small fiber neuropathy. The clinical data on sudomotor function testing indicates that it may be the most sensitive method for detecting small fiber peripheral neuropathy, which could signal the onset of peripheral distal neuropathy [22].

\subsection{TBL-ABI Assessment}

The TBL-ABI comprises of four Bluetooth blood pressure devices using the patented pulse wave measurement of the blood pressure and volume plethysmography. Blood pressure measurements are performed at each arm, as well as at the posterior tibial artery and dorsalis pedis artery of each ankle. The left and right ankle brachial measurements are based on the ratio of the lowest ankle systolic pressure and the highest arm systolic pressure, allowing the volume plethysmography to be displayed for each ankle to evaluate the arterial blood flow.

Similarly, the volume plethysmography analysis provides markers for estimating the Central Aortic Systolic Pressure (CASP), as shown in Figure 5 [23].

As can be seen from the graph above, CASP values are obtained via the cuff-based volume plethysmography analysis at the brachial artery using the expression CASP $=($ Systolic Pressure $\times$ pAIx $) / 100$.

Based on the available evidence, Ankle Brachial Index (ABI) is considered normal if within the $1.0-1.30$ range, whereby pre-compressible range is indicated by $1.31-1.39$, acceptable range spans from 0.91 to 0.99 , borderline range is defined as $0.90-0.81$, defined range by $0.61-0.80$, severe range by $\leq 0.60$, and noncompressible range by $\geq 1.39$ [24]. Even though the ABI was originally developed for the noninvasive diagnosis of lower-extremity Peripheral Artery Disease (PAD), it has since been used to indicate atherosclerosis at different vascular sites. Furthermore, the ABI has found clinical applications as a prognostic marker of cardiovascular impairment and cardiovascular events in patients not presenting with any PAD symptoms [25].

\subsection{TM Flow Clinical Studies}

The TM Flow markers have been evaluated with clinical studies in the US, India, and Brazil.

Below, is the summary of the clinical study results.

1) Stress Index marker is correlated with the findings yielded by CRP lab test at $\rho=0.40, \mathrm{p}<0.0001[13]$;

2) The Sweat Peak sudomotor response marker correlates inversely with the severity of the symptoms measured by the peripheral neuropathy scale [13];

3) PTG VLFi marker is correlated with the findings yielded by fibrinogen lab test at $\rho=0.43, \mathrm{p}<0.0001[13]$; 
Central Aortic Pressure Waveform

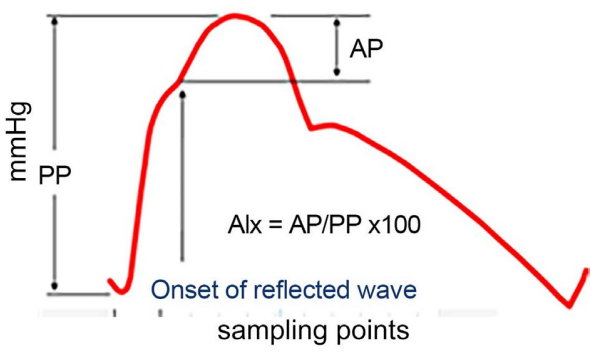

Figure 5. CASP estimation.

4) PTGi marker is suitable for detecting coronary heart disease using coronary angiography, as it is characterized by high sensitivity (86.1\%) and specificity (87.2\%) [14];

5) The area under the ROC curve (AUC) $=0.929$ indicates that the PTG-TP cutoff value 406.2 should be adopted to obtain a sufficiently high sensitivity (95.7\%) and specificity (84.4\%) when identifying insulin resistance [16].

\section{Discussion}

The pathophysiology of the endothelial cells has been the subject of extensive research [26] [27] [28], suggesting that the presence of underlying conditions (e.g., hypertension, cardiovascular disease, diabetes, endothelial cell infection and/or dysfunction), as well as older age typically result in more severe COVID-19 outcomes [26].

As such comorbidities are often associated with endothelial cell dysfunction or damage [28], this should be considered when treating COVID-19 patients. Moreover, ample body of evidence correlates COVID-19-related deaths to pulmonary edema, Acute Respiratory Distress Syndrome (ARDS), cytokine storm, diffuse coagulation, and organ failure [29] [30] [31], while ARDS is considered to result from pulmonary EC damage [32] [33].

The endothelial dysfunction or endothelial cell impairment causes an insufficient release Nitric Oxide (NO), which in turn leads to vessel constriction [32]. As nitric oxide deficiency is common in COVID-19 patients [33], it may cause contraction of smooth muscles, thereby reducing the capability to neutralize ROSand NO-mediated antiviral load [34] [35]. Given that endothelial cell function regulates coagulation and acts as a counterbalance for thrombin, when impaired, it prohibits a correct response to viral infection by improperly activating the coagulation pathway, thereby causing an anticoagulation imbalance [34]. This mechanism is increasingly being linked to endothelial dysfunction, inflammation, vascular leakage, and anticoagulation imbalance in COVID-19 patients [29]-[35], which are further exacerbated by the adverse impact of the virus on the autonomic nervous system [37].

Indeed, COVID-19 is believed to result from the activation of the sympathetic system which causes a pro-inflammation cytokine release [36] [37] [38]. As vagal stimulation results in an anti-inflammatory response [39], it may be a promising 
target in the treatment of autonomic nervous system impairments [40]. On the other hand, the autonomic dysfunction in COVID-19 patients could be caused by the virus itself [40] [41] [42] [43]. Moreover, autonomic disorders such as $\mathrm{OH}$ and postural orthostatic tachycardia syndrome (POTS) have been linked to autoantibodies [43] [44] such as $\alpha$-/ $\beta$-adrenoceptors and muscarinic receptors [45] [46], which commonly precede infections in POTS [44] as well as provide a link between autoimmune biomarkers and autoimmune disorders [47]. As in COVID-19 patients, autonomic dysfunction (such as orthostatic hypotension, POTS, and small fiber neuropathy) can occur prior or after the infection period [43] [44] [45] [46] [47], it should be assessed at intake.

Since the TM Flow system is already being used by physicians for early detection of chronic diseases involving endothelial and autonomic nervous system impairments, it would be helpful in the early identification of individuals who are at risk of suffering from severe COVID-19 complications, allowing appropriate treatments to be initiated in a timely manner, and potentially reducing hospitalizations and deaths.

Of course, the TM Flow and Lifeprobes kiosk does not diagnose or treat the COVID-19. Specifically, the TM Flow system and Lifeprobes Kiosk could be useful in patient triage. By assessing the endothelial function, vital signs, Ankle Brachial Index measurements and diagnosing Cardiac Autonomic Neuropathy, it potentially detects the Patients at risk.

When using TM Flow results for triage, the following criteria should be applied:

- Two or more abnormal endothelial markers are indicative of abnormal endothelial dysfunction;

- Two or more abnormal Cardiac Autonomic Reflex tests are indicative of defined Cardiac Autonomic Neuropathy and abnormal Autonomic Nervous System function;

- High Central Aortic Systolic Pressure (CASP) according to the age;

- Ankle brachial index less or equal at 0.8 .

Once the aforementioned criteria have been applied, patients with at least one abnormal criterion are at risk and the physician will be able to decide if the patient should receive the FDA approved [48] treatments or those that have received Emergency Use Authorization (EUA) [49] for reducing the risk of COVID19 complications.

The TM Flow system can also be used to identify "Long COVID" as it allows early detection of autonomic nervous system disorders, such as CAN, OH, POTS, and small fiber neuropathy.

If indicated, physician will be able decide whether the patient needs available pharmacologic or non-pharmacologic treatments, such as those indicated below:

- Non-pharmacologic treatment for $\mathrm{OH}$ is based on exercise, fluid intake, and salt repletion, while patients should be advised to avoiding any exacerbating factors, such as isometric exercises and compression garments [7];

For small fiber neuropathy, alpha-lipoic acid appears to cause a delay or even 
reverse the effects seen in peripheral diabetic neuropathy due to its antioxidant properties. Evidence yielded by clinical trials suggests that $600 \mathrm{mg}$ of alpha-lipoic acid daily for one month improves neuropathic deficits in majority of patients [50] [51];

- Pharmacologic treatments for POST typically involve discontinuation of norepinephrine reuptake inhibitors such as duloxetine, nortryptiline and tapentadol [52];

- Pharmacologic treatments for $\mathrm{OH}$ include fludrocortisone (a fluid expander), it can be used if hypovolemia is a dominant symptom. However, as it is associated with considerable side-effects and is not particularly well tolerated, patients need to be carefully monitored for fluid retention and hypokalemia [53]. On the other hand, midodrine (a sympathomimetic $\alpha$-1-agonist) increases vasoconstriction and venous return to the heart and can effectively treat orthostatic hypotension and tachycardia in patients who have a baseline normal or low-normal blood pressure. If midodrine is prescribed, patients should be advised to avoid the supine position as much as possible to prevent excessive blood pressure increase. Moreover, male patients with prostatic problems may experience urinary retention, while scalp itch is a common side-effect in patients of both sexes [53]. For patients suffering from small fiber neuropathy, antidepressants, anti-seizure medications, or analgesics could be prescribed to treat painful sensory paresthesia [54].

\section{Conclusion}

The TM Flow system and Lifeprobes Kiosk are valuable tools that can be employed in clinical settings for conducting non-invasive and fast tests with instantaneous results. As such, they can be employed in the triage of COVID-19 patients, as those deemed at risk of complications can be offered timely treatment, thus reducing morbidity and mortality rates. The TM Flow system also helps diagnose "Long COVID" which is believed to arise from autonomic nervous system disorders and small fiber neuropathy.

\section{Acknowledgements}

The author wishes to thank all individuals who provided their contributions to the spectral analysis and sudomotor marker clinical investigations.

\section{Conflicts of Interest}

The author reports no conflict of interest in any clinical investigation, as the manuscript merely provides a review of already published data obtained by other researchers.

\section{References}

[1] Palayew, A., Norgaard, O., Safreed-Harmon, K., Andersen, T.H., Rasmussen, L.N. and Lazarus, J.V. (2020) Pandemic Publishing Poses a New COVID-19 Challenge. 
Nature Human Behaviour, 4, 666-669. https://doi.org/10.1038/s41562-020-0911-0

[2] Wang, L., Wang, Y., Ye, D. and Liu Q. (2020) A Review of the 2019 Novel Coronavirus (COVID-19) Based on Current Evidence. International Journal of Antimicrobial Agents, 55, Article ID: 105948.

https://doi.org/10.1016/j.ijantimicag.2020.105948

[3] https://www.euro.who.int/en/health-topics/health-emergencies/coronavirus-covid19/statements/statement-older-people-are-at-highest-risk-from-covid-19,-but-all-m ust-act-to-prevent-community-spread

[4] CDC (Centers for Disease Control and Prevention) (2021) Evidence for Conditions that Increase Risk of Severe Illness.

https://www.cdc.gov/coronavirus/2019-ncov/science/science-briefs/underlying-evid ence-table.html

[5] CDC (Centers for Disease Control and Prevention) (2021) COVID-19 Vaccines and Vaccination.

https://www.cdc.gov/coronavirus/2019-ncov/science/science-briefs/fully-vaccinated -people.html

[6] Jin, Y., Ji, W., Yang, H., Chen, S., Zhang, W. and Duan, G. (2020) Endothelial Activation, and Dysfunction in COVID-19: From Basic Mechanisms to Potential Therapeutic Approaches. Signal Transduction and Targeted Therapy, 5, Article No. 293. https://doi.org/10.1038/s41392-020-00454-7

[7] Nägele, M.P., Haubner, B., Tanner, F.C., Ruschitzka, F. and Flammer, A.J. (2020) Endothelial Dysfunction in COVID-19: Current Findings and Therapeutic Implications. Atherosclerosis, 314, 58-62. https://doi.org/10.1016/j.atherosclerosis.2020.10.014

[8] Leitzke, M., Stefanovic, D., Meyer, J.-J., Schimpf, S. and Schönknecht, P. (2020) Autonomic Balance Determines the Severity of COVID-19 Courses. Bioelectronic Medicine, 6, Article No. 22. https://doi.org/10.1186/s42234-020-00058-0

[9] Dani, M., Dirksen, A., Taraborrelli, P., Torocastro, M., Panagopoulos, D., Sutton, R. and Lim, P.B. (2021) Autonomic Dysfunction in 'Long COVID': Rationale, Physiology, and Management Strategies. Clinical Medicine, 21, e63-e67.

https://doi.org/10.7861/clinmed.2020-0896

[10] Elgendi, M., Norton, I., Brearley, M., Abbott, A. and Schuurmans, D. (2014) Detection of $A$ and $B$ Waves in the Acceleration Photoplethysmogram. BioMedical Engineering OnLine, 13, Article No. 139. https://doi.org/10.1186/1475-925X-13-139

[11] Takazawa, K., Tanaka, N., Fujita, M., Matsuoka, O., Saiki, T., Aikawa, M., et al. (1998) Assessment of Vasoactive Agents and Vascular Aging by the Second Derivative of Photoplethysmogram Waveform. Hypertension, 32, 365-370.

https://doi.org/10.1161/01.HYP.32.2.365

[12] Maklin, C. (2019) Fast Fourier Transform. https://towardsdatascience.com/fast-fourier-transform-937926e591cb

[13] Lewis, J.E., Atlas, S.E., Rasul, A., Farooqi, A., Lantigua, L., Higuera, O.L., et al. (2017) New Method of Sudomotor Function Measurement to Detect Microvascular Disease and Sweat Gland Nerve or Unmyelinated C Fiber Dysfunction in Adults with Retinopathy. Journal of Diabetes \& Metabolic Disorders, 16, Article No. 26. https://doi.org/10.1186/s40200-017-0307-5

[14] Gandhi, P.G. and Rao, G.H.R. (2014) The Spectral Analysis of the Photoplethysmography to Evaluate an Independent Cardiovascular Risk Factor. International Journal of General Medicine, 7, 539-547. https://doi.org/10.2147/IJGM.S70892

[15] Muniyappa, R., Lantorno, M. and Quon, M.J. (2008) An Integrated View of Insulin Resistance and Endothelial Dysfunction. Endocrinology and Metabolism Clinics of 
North America, 37, 685-711. https://doi.org/10.1016/j.ecl.2008.06.001

[16] De Souza, A.L., Batista, G.A. and Alegre, S.M. (2017) Assessment of Insulin Sensitivity by the Hyperinsulinemic Euglycemic Clamp: Comparison with the Spectral Analysis of Photoplethysmography. Journal of Diabetes and Its Complications, 31, 128-133. https://doi.org/10.1016/j.jdiacomp.2016.10.018

[17] Serhiyenko, V.A. and Serhiyenko, A.A. (2018) Cardiac Autonomic Neuropathy: Risk Factors, Diagnosis, and treatment. World Journal of Diabetes, 9, 1-24. https://doi.org/10.4239/wjd.v9.i1.1

[18] Spallone, V., Ziegler, D., Freeman, R., Bernardi, L., Frontoni, S., Pop-Busui, R., Stevens, M., Kempler, P., Hilsted, J., Tesfaye, S., et al. (2011) Cardiovascular Autonomic Neuropathy in Diabetes: Clinical Impact, Assessment, Diagnosis, and Management. Diabetes/Metabolism Research and Reviews, 27, 639-653. https://doi.org/10.1002/dmrr.1239

[19] Dimitropoulos, G., Tahrani, A.A. and Stevens, M.J. (2014) Cardiac Autonomic Neuropathy in Patients with Diabetes Mellitus. World Journal of Diabetes, 5, 17-39. https://doi.org/10.4239/wjd.v5.i1.17

[20] Yun, J.-S., Kim, J.-H., Song, K.-H., Ahn, Y.-B., Yoon, K.-H., Yoo, K.-D., Park, Y.-M. and Ko, S.-H. (2014) Cardiovascular Autonomic Dysfunction Predicts Severe Hypoglycemia in Patients with Type 2 Diabetes: A 10-Year Follow-Up Study. Diabetes Care, 37, 235-241. https://doi.org/10.2337/dc13-1164

[21] Yun, J.S., Park, Y.M., Cha, S.A., Ahn, Y.B. and Ko, S.H. (2018) Progression of Cardiovascular Autonomic Neuropathy and Cardiovascular Disease in Type 2 Diabetes. Cardiovascular Diabetology, 17, Article No. 109. https://doi.org/10.1186/s12933-018-0752-6

[22] Low, V.A., Sandroni, P., Fealey, R.D. and Low, P.A. (2006) Detection of Small-Fiber Neuropathy by Sudomotor Testing. Muscle \& Nerve, 34, 57-61. https://doi.org/10.1002/mus.20551

[23] Narayan, O., Casan, J., Szarski, M., Dart, A.M., Meredith, I.T. and Cameron, J.D. (2014) Estimation of central Aortic Blood Pressure: A Systematic Meta-Analysis of Available Techniques. Journal of Hypertension, 32, 1727-1740.

https://doi.org/10.1097/HJH.0000000000000249

[24] Gerhard-Herman, M.D., Gornik, H.L., Barrett, C., Barshes, N.R., Corriere, M.A., Drachman, D.E., et al. (2016) 2016 AHA/ACC Guideline on the Management of Patients with Lower Extremity Peripheral Artery Disease: Executive Summary: A Report of the American College of Cardiology/American Heart Association Task Force on Clinical Practice Guidelines. Circulation, 135, Article No. e790. https://doi.org/10.1161/CIR.0000000000000501

[25] Olin, J.W. and Sealove, B.A. (2010) Peripheral Artery Disease: Current Insight into the Disease and Its Diagnosis and Management. Mayo Clinic Proceedings, 85, 678-692. https://doi.org/10.4065/mcp.2010.0133

[26] Sturtzel, C. (2017) Endothelial Cells. In: Sattler, S. and Kennedy-Lydon, T., Eds., The Immunology of Cardiovascular Homeostasis and Pathology, Vol. 1003, Springer International Publishing, Cham, 71-91. https://doi.org/10.1007/978-3-319-57613-8 4

[27] Aird, W.C. (2007) Phenotypic Heterogeneity of the Endothelium: II. Representative Vascular Beds. Circulation Research, 100, 174-190. https://doi.org/10.1161/01.RES.0000255690.03436.ae

[28] Pober, J.S. and Sessa, W.C. (2007) Evolving Functions of Endothelial Cells in Inflammation. Nature Reviews Immunology, 7, 803-815. 
https://doi.org/10.1038/nri2171

[29] Teuwen, L.A., Geldhof, V., Pasut, A. and Carmelie, P. (2020) COVID-19: The Vasculature Unleashed. Nature Reviews Immunology, 20, 389-391.

https://doi.org/10.1038/s41577-020-0343-0

[30] Green, S.J. (2020) Covid-19 Accelerates Endothelial Dysfunction and Nitric Oxide Deficiency. Microbes and Infection, 22, 149-150. https://doi.org/10.1016/j.micinf.2020.05.006

[31] Akerstrom, S., Gunalan, V., Keng, C.T., Tan, Y.J. and Mirazimi, A. (2009) Dual Effect of Nitric Oxide on SARS-CoV Replication: Viral RNA Production and Palmitoylation of the S Protein Are Affected. Virology, 395, 1-9. https://doi.org/10.1016/j.virol.2009.09.007

[32] Akerström, S., Mousavi-Jazi, M., Klingström, J., Leijon, M., Lundkvist, A. and Mirazimi, A. (2005) Nitric Oxide Inhibits the Replication Cycle of Severe Acute Respiratory Syndrome Coronavirus. Journal of Virology, 79, 1966-1969.

https://doi.org/10.1128/JVI.79.3.1966-1969.2005

[33] Panigada, M., Bottino, N., Tagliabue, P., Grasselli, G., Novembrino, C., Chantarangkul, V., et al. (2020) Hypercoagulability of COVID-19 Patients in Intensive Care Unit: A Report of Thromboelastographic Findings and Other Parameters of Hemostasis. Journal of Thrombosis and Haemostasis, 18, 1738-1742. https://doi.org/10.1111/jth.14850

[34] Ranucci, M., Ballotta, A., Di Dedda, U., Baryshnikova, E., Poli, M.D., Resta, M., et al. (2020) The Procoagulant Pattern of Patients with COVID-19 Acute Respiratory Distress Syndrome. Journal of Thrombosis and Haemostasis, 18, 1747-1751. https://doi.org/10.1111/jth.14854

[35] Iba, T., Levy, J.H., Levi, M., Connors, J.M. and Thachil, J. (2020) Coagulopathy of Coronavirus Disease 2019. Critical Care Medicine, 48, 1358-1364. https://doi.org/10.1097/CCM.0000000000004458

[36] Goldstein, D.S. (2020) The Extended Autonomic System, Dyshomeostasis, and COVID19. Clinical Autonomic Research, 30, 299-315. https://doi.org/10.1007/s10286-020-00714-0

[37] Fudim, M., Qadri, Y.J., Ghadimi, K., MacLeod, D.B., Molinger, J., Piccini, J.P., et al. (2020) Implications for Neuromodulation Therapy to Control Inflammation and Related Organ Dysfunction in COVID-19. Journal of Cardiovascular Translational Research, 13, 894-899. https://doi.org/10.1007/s12265-020-10031-6

[38] Konig, M.F., Powell, M., Staedtke, V., Bai, R.Y., Thomas, D.L., Fischer, N., et al. (2020) Preventing Cytokine Storm Syndrome in COVID-19 Using a-1 Adrenergic Receptor Antagonists. Journal of Clinical Investigation, 137, 3345-3347. https://doi.org/10.1172/JCI139642

[39] Staedtke, V., Bai, R.-Y., Kim, K., Darvas, M., Davila, M.L., Riggins, G.J., et al. (2018) Disruption of a Self-Amplifying Catecholamine Loop Reduced Cytokine Release Syndrome. Nature, 564,273-277. https://doi.org/10.1038/s41586-018-0774-y

[40] Guilmot, A., Slootjes, S.M., Sellimi, A., Bronchain, M., Hanseeuw, B., Belkhir, L., et al. (2020) Immune-Mediated Neurological Syndromes in SARS-CoV-2-Infected Patients. Journal of Neurology, 268, 751-757.

https://doi.org/10.1007/s00415-020-10108-x

[41] Ruzieh, M., Batizy, L., Dasa, O., Oostra, C. and Grubb, B. (2017) The Role of Autoantibodies in the Syndromes of Orthostatic Intolerance: A Systematic Review. Scandinavian Cardiovascular Journal, 51, 243-247. https://doi.org/10.1080/14017431.2017.1355068 
[42] Li, H., Kem, D.C., Reim, S., Khan, M., Vanderlinde-Wood, M., Zillner, C., et al. (2012) Agonistic Autoantibodies as Vasodilators in Orthostatic Hypotension: A New Mechanism. Hypertension, 59, 402-408. https://doi.org/10.1161/HYPERTENSIONAHA.111.184937

[43] Fedorowski, A., Li, H., Yu, X., Koelsch, K.A., Harris, V.M., Liles, C., et al. (2017) Antiadrenergic Autoimmunity in Postural Tachycardia Syndrome. EP Europace, 19, 1211-1219. https://doi.org/10.1093/europace/euw154

[44] Li, H., Yu, X., Liles, C., Khan, M., Vanderlinde-Wood, M., Galloway, A., et al. (2014) Autoimmune Basis for Postural Tachycardia Syndrome. Journal of the American Heart Association, 3, Article ID: e000755. https://doi.org/10.1161/JAHA.113.000755

[45] Yu, X., Stavrakis, S., Hill, M.A., Huang, S., Reim, S., Li, H., et al. (2012) Autoantibody Activation of Beta-Adrenergic and Muscarinic Receptors Contributes to an "Autoimmune" Orthostatic Hypotension. Journal of the American Society of Hypertension, 6, 40-47. https://doi.org/10.1016/j.jash.2011.10.003

[46] Watari, M., Nakane, S., Mukaino, A., Nakajima, M., Mori, Y., Maeda, Y., et al. (2018) Autoimmune Postural Orthostatic Tachycardia Syndrome. Annals of Clinical and Translational Neurology, 5, 486-492. https://doi.org/10.1002/acn3.524

[47] Blitshteyn, S., Brinth, L., Hendrickson, J.E. and Martinez-Lavin, M. (2018) Autonomic Dysfunction, and HPV Immunization: An Overview. Immunologic Research, 66, 744754. https://doi.org/10.1007/s12026-018-9036-1

[48] https://www.fda.gov/drugs/emergency-preparedness-drugs/coronavirus-covid-19-d rugs\#: :text=Veklury\%20(Remdesivir)\%20is,\%2D19\%20requiring\%20hospitalization

[49] https://www.fda.gov/emergency-preparedness-and-response/mcm-legal-regulatoryand-policy-framework/emergency-use-authorization\#coviddrugs

[50] Shouman, K., Vanichkachorn, G., Cheshire, W.P., Suarez, M.D., Shelly, S., Lamotte, G.J., Sandroni, P., Benarroch, E.E., Berini, S.E., Cutsforth-Gregory, J.K., Coon, E.A., Mauermann, M.L., Low, P.A. and Singer, W. (2021) Autonomic dysfunction Following COVID-19 Infection: An Early Experience. Clinical Autonomic Research, 31, $385-$ 394. https://doi.org/10.1007/s10286-021-00803-8

[51] Vallianou, N., Evangelopoulos, A. and Koutalas, P. (2009) Alpha-Lipoic Acid and Diabetic Neuropathy. Review of Diabetic Studies, 6, 230-236. https://doi.org/10.1900/RDS.2009.6.230

[52] Sheldon, R.S., Grubb II, B.P., Olshansky, B., Shen, W.K., Calkins, H., Brignole, M., et al. (2015) 2015 Heart Rhythm Society Expert Consensus Statement on the Diagnosis and Treatment of Postural Tachycardia Syndrome, Inappropriate Sinus $\mathrm{Ta}$ chycardia, and Vasovagal Syncope. Heart Rhythm, 12, e41-e63. https://doi.org/10.1016/j.hrthm.2015.03.029

[53] Freeman, R., Abuzinadah, A.R., Gibbons, C., Jones, P., Miglis, M.G. and Sinn, D.I. (2018) Orthostatic Hypotension: JACC State-of-the-Art Review. Journal of the American College of Cardiology, 72, 1294-1309. https://doi.org/10.1016/j.jacc.2018.05.079

[54] https://www.hopkinsmedicine.org/neurology neurosurgery/centers clinics/periphe ral nerve/conditions/small fiber sensory neuropathy.html 